\title{
Inferring 3D Kinematics of Carpal Bones from Single View Fluoroscopic Sequences
}

\author{
Xin Chen ${ }^{1}$, Jim Graham ${ }^{1}$, Charles Hutchinson ${ }^{2}$, and Lindsay Muir ${ }^{3}$ \\ 1 ISBE, School of Cancer and Enabling Sciences, University of Manchester, Oxford Road, \\ Manchester, M13 9PT, UK \\ \{xin.chen, jim.graham\} @manchester.ac.uk \\ 2 Clinical Sciences Research Institute, Clinical Sciences Building, University Hospital \\ -Walsgrave Campus, Clifford Bridge Road, Coventry, CV2 2DX, UK \\ 3 Consultant Orthopaedic Surgeon, Salford Royal Hospital NHS Foundation Trust, Stott Lane, \\ Salford, M6 8HD, UK
}

\begin{abstract}
We present a novel framework for inferring 3D carpal bone kinematics and bone shapes from a single view fluoroscopic sequence. A hybrid statistical model representing both the kinematics and shape variation of the carpal bones is built, based on a number of 3D CT data sets obtained from different subjects at different poses. Given a fluoroscopic sequence, the wrist pose, carpal bone kinematics and bone shapes are estimated iteratively by matching the statistical model with the 2D images. A specially designed cost function enables smoothed parameter estimation across frames. We have evaluated the proposed method on both simulated data and real fluoroscopic sequences. It was found that the relative positions between carpal bones can be accurately estimated, which is potentially useful for detection of conditions such as scapholunate dissociation.
\end{abstract}

Keywords: Carpal bones kinematics, 2D 3D registration, Statistical model.

\section{Introduction}

Chronic pain in the wrist arises due to a number of conditions, such as instability patterns, nonunion or malunion of fractures, primary osteoarthritis and inflammatory arthritis. The result for patients is a severe reduction in quality of life due to impairment of everyday functions, lost work time, increased morbidity and loss of the capacity to live independently. The current method of distinguishing between these conditions is by examining 2D video fluoroscopy sequences showing movement of the hand from full ulnar to full radial deviation and from full flexion to extension in two orthogonal views. From these images clinicians can infer the three-dimensional translations and rotations of the carpal bones that take place during wrist movement, and arrive at a differential diagnosis on the basis of variations from normal bone kinematics. The interpretation is difficult and the accuracy of diagnosis depends wholly on the experience of the practitioner. Currently, accurate diagnosis requires referral to a specialist hand consultant and treatment is often delayed to the detriment of the patient.

The aim of the project is computer interpretation of the fluoroscopy sequences to attain a higher degree of objectivity and quantification in the diagnostic process. During 
wrist movement, the eight carpal bones follow a complex, multi-dimensional trajectory, making interpretation of radiographs difficult. For this study we have trained a hybrid statistical model (SM) from a set of CT images from different subjects at different poses. Subsequently, the full 3D carpal bone motions can be recovered by matching the SM with the fluoroscopy sequences through 3D-2D image registration techniques. A number of studies have sought to represent the carpal kinematics using CT or MR data, mainly concentrating on representing 'average' kinematics over a small number of individuals (e.g. [1], [2]). More recently, Van deGiessen et al. [3] presented a 3D rigid registration method based on segmented meshes, which aims to build SM of carpal bones. A study of carpal bone kinematics based on a 4D imaging system was reported in [4]. 3D-2D registration has been the subject of many studies (e.g. [5]), mainly in the field of registration of pre-operative $\mathrm{MR}$ or CT images to intra-operative 2D images. Our work differs from the above in that we seek to achieve registration of a $2 \mathrm{D}$ image sequence to a 3D model (not derived from the same individual) to derive the kinematics of an individual wrist. Zheng [6] took a similar approach to estimate the orientation of pelvis from a single $\mathrm{X}$-ray image.

The main contributions of this paper, distinguishing it from these studies, are: (1) A hybrid SM is developed representing both the complex kinematics and shape variation of the eight carpal bones plus radius and ulna. (2) The full 3D motion and bone shapes are recovered by matching the SM with a single view fluoroscopy sequence: a difficult ill-posed problem. (3) Our initial results show that the relative positions between the carpal bones can be estimated accurately through the proposed framework. We are not aware of any study which attempts to make a $2 \mathrm{D}$ to $3 \mathrm{D}$ inference in a system of this level of complexity.

The system consists of a training phase and a 3D-2D image registration phase. We currently have CT data from 10 subjects, each at five poses (neutral pose and two extreme poses in flexion-extension and radial-ulnar deviation). In the training phase, only the data from the neutral pose and two extreme poses in the radial-ulnar movement were used, as the radial-ulnar movement is the current concern of this paper. The segmentation of each bone and rigid registration parameters that align bones at different poses within and across the subjects were obtained using an iterative segmentation and registration algorithm [7]. A hybrid statistical model, representing both the kinematics and shape variation, was built efficiently from the results of the segmentation-registration framework. The kinematic model was built based on the transformation parameters, while the segmentation result was used to build the statistical shape model for each individual bone. In the 3D-2D image registration phase, the 3D rigid transformation, the kinematic motion and bone shapes were estimated in sequence from each frame of the fluoroscopy sequences. Detailed descriptions are given in the following sections.

\section{Problem Parameterisation}

We use a perspective projection model to represent the relationship between the $2 \mathrm{D}$ fluoroscopy image and the 3D configuration of bones. Almost all parameters necessary for this model (pixel size and optical centre) are known. The distance from the X-ray source and the detector needs to be measured for each patient. If this parameter is not 
accurate, it will lead to a scale difference of the estimated 3D model. The resulting translation effects on the relative motion between carpal bones at pixels away from the centre of the field is very small.

Three sets of parameters need to be estimated during image registration in order to interpret the true 3D motion of the carpal bones: (1) Rigid transformation parameters of the wrist and a global scale factor, denoted by $\theta=\left\{t_{x}, t_{y}, t_{z}, \alpha, \beta, \gamma, s_{\text {global }}\right\} . t_{x}, t_{y}$ and $t_{z}$ denote the translations, and $\alpha, \beta$ and $\gamma$ denote the rotation angles. $s_{\text {global }}$ controls the distance between the centroid of each bone to the origin in the radius, and the global size of the bones. (2) Kinematic model parameters $M$ representing the carpal bone poses during movement. (3) Shape model parameters $Q_{i}$ and scale factor $s_{i}$ for each bone $(i)$.

\section{Training of Kinematic Model and Shape Model}

We use the six rigid transformation parameters for each bone to train the kinematic model. The common coordinate system for all pose and scale parameters has an origin at the centroid of the head of radius for one subject. The pose of one subject is described by $\left(t x_{1}, t y_{1}, t z_{1}, \alpha_{1}, \beta_{1}, \gamma_{1}, \ldots, t x_{10}, t y_{10}, t z_{10}, \alpha_{10}, \beta_{10}, \gamma_{10}\right)^{t} .(8$ carpal bones, 1 radius and 1 ulna). The orientation parameters all occupy values distant from the angular discontinuity. Then the kinematic model can be parameterised as,

$$
M=\mu^{m}+\phi^{m} b^{m}
$$

where the mean pose $\mu^{m}$ ( $m$ is a notation indicating the model parameters) and the principal subspace matrix $\phi^{m}$ are computed from 3 (poses) $\times 10$ (subjects) training samples using PCA. The vector $b^{m}$ represents the kinematic parameters that describe the pose of $M$ along each principal direction. In our experiments, the first 8 significant modes are used, which keeps $98 \%$ of variation.

The statistical shape model of each bone is a point distribution model, built using the segmented volume of the same training subjects. The 3D structure of each bone is described by a set of approximately 1000 points on the segmented surface. Correspondence between these points across subjects was established by the minimum description length algorithm [8]. The deformable shape model is then described as,

$$
Q_{i}=\mu_{i}^{q}+\phi_{i}^{q} b_{i}^{q}
$$

where $\mu_{i}^{q}$ and $\phi_{i}^{q}$ ( $q$ is a notation indicating the shape parameters) are the mean shape and the principal subspace matrix for the $i^{t h}$ bone. $b_{i}^{q}$ is the shape model parameter to be estimated. In order to keep the complexity within limits, only the first 3 significant modes are used which keeps $84 \%$ of variation.

Based on the point distribution model of each bone and the kinematic model, a hybrid statistical mesh model can be built by using the Crust mesh construction algorithm [9]. Figure 1 shows the poses of the first mode of the kinematic model (represented by the mean shapes of each bone) and the shapes of the first mode of the scaphoid.

\section{3D-2D Image Registration}

The statistical mesh model from the training data is then used to match with the fluoroscopic sequence to infer the 3D motion and bone shapes. Figure 2 (a) summarises 

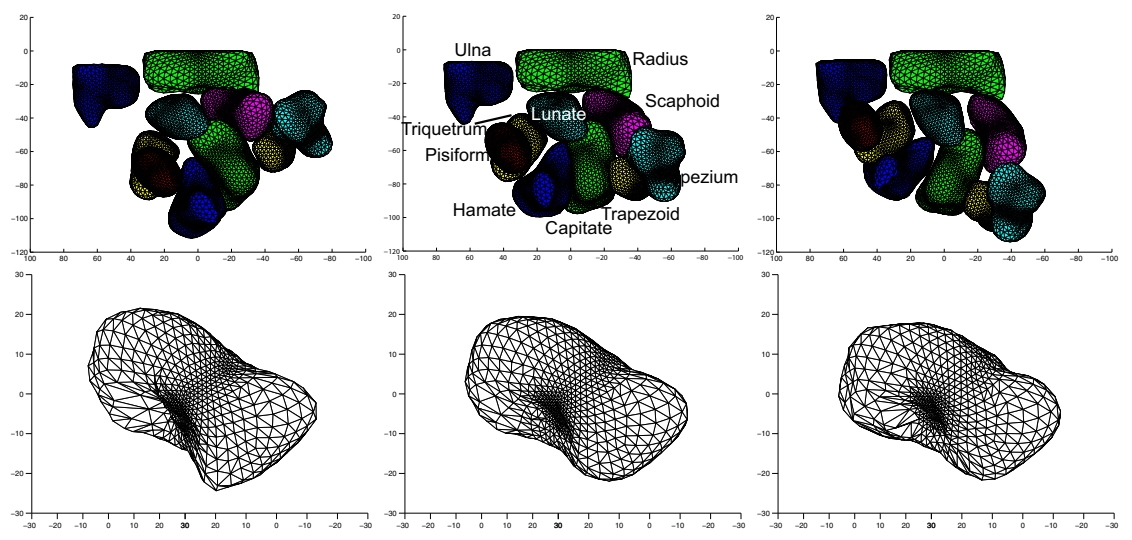

Fig. 1. Top row: The poses of the first mode of kinematic model. Bottom row: the first mode of the shape model of the scaphoid. In each case the mean $+/-1.5$ s.d. are shown.

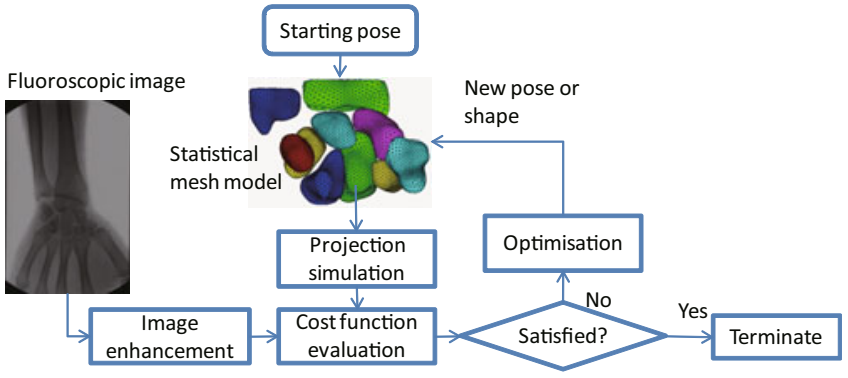

(a)

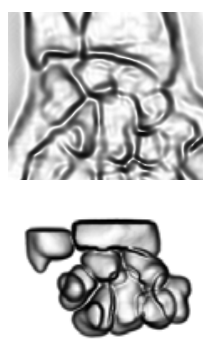

(b)

Fig. 2. Overview of the 3D-2D image registration process. (b) The gradient magnitude map of the fluoroscopic image after enhancement (cropped to show the region of interest) (top) and the simulated image from mesh model (bottom).

the registration process, in which the preprocessed fluoroscopic image is iteratively matched with a simulated projection generated from an updated pose of the mesh model. Detailed descriptions are given in the following subsections.

\subsection{Fluoroscopic Image Enhancement and Projection Simulation}

As the edges are strong features that can be used for image matching, the fluoroscopic image was firstly pre-processed to enhance the edges and reduce noise in homogenous regions. Local intensity normalisation was achieved by subtraction of the local mean intensity and division by the local standard deviation. The anisotropic diffusion [10] filter is then used to smooth the image while preserving the edges. Figure 2(b) shows an example of the gradient magnitude map of the fluoroscopic image after enhancement.

To optimise the pose parameters we iteratively generate projections from the mesh model with updated parameters, using the perspective projection described in section 
2 The mesh model is considered to be a binary volume, and the projected intensity is negatively proportional to the sum of binary values along the ray from the source to each pixel in the image plane. Figure 2 (b) shows an example.

\subsection{Cost Function}

To evaluate the similarity between the fluoroscopic image and the simulated image, we investigated several forms of the cost function, achieving best results from the one shown in Eqn. (3), based on the gradient along horizontal and vertical directions as well as the gradient magnitude of the two images. Additionally, the adjacent frames of the current fluoroscopic image were also taken into account in the cost function to make the estimated poses smooth across frames.

Taking C(A,B) as the Normalised Correlation Coefficient between two images $A$ and $B$, we can write the cost function as:

$$
E=C\left(O m_{k-1}, O m_{k}\right)+\sum_{p=k-1, k, k+1} w_{p}\left(C\left(\operatorname{Im}_{p}, D m_{k}\right)+C\left(I x_{p}, D x_{k}\right)+C\left(I y_{p}, D y_{k}\right)\right)
$$

where $k$ is the current frame number of the fluoroscopic sequence. $I m_{p}, I x_{p}$ and $I y_{p}$ are the gradient magnitude image, vertical gradient and horizontal gradient of the fluoroscopic image at the $p^{t h}$ frame respectively. $D m_{k}, D x_{k}$ and $D y_{k}$ are the corresponding values of the simulated image. The second term calculates a cross-correlation between sets of three adjacent frames with weights $w_{k-1}, w_{k}$ and $w_{k+1}=0.2,0.6,0.2$ respectively, making the estimated pose smooth across frames. For the first term of the cost function, the vertices in the statistical mesh model are projected to the image plane, we assume the intensities at those projected points are similar across adjacent frames. $O m_{k-1}$ and $\mathrm{Om}_{k}$ represent the gradient magnitude of the previous frame and the current frame at the projected correspondence positions. The first term makes the shape of the cost function sharper, leading to a faster and more accurate optimisation result. The $(k-1)^{\text {th }}$ frame and $(k+1)^{t h}$ frame are not evaluated for the first and last frame respectively.

\subsection{Optimisation}

The optimisation method used is the best neighbour search combined with parabola fitting. The multi-dimensional search space $(\theta, M$ and $Q)$ is explored by iteratively individual 1D line search. The cost function is evaluated at the current position, positive and negative neighbour positions (defined by a search range), then an optimum is found by fitting a parabola to the 3 evaluated positions. The optimum is iteratively refined by reducing the search range until convergence.

In our case, the true sizes of the bones are unknown; recovering the $3 \mathrm{D}$ pose from a single image is therefore a difficult, ill posed, problem. Any movement along the outof-plane translation, could be compensated by scaling of the bone. In order to minimise this effect, the optimisation is carefully sequenced. We firstly assume that the wrist is not moving along the out-of-plane direction during radial-ulnar movement $\left(t_{y}=0\right)$, as it is placed on a flat surface. The position of the model is firstly initialised by clicking the centre of the radius in the first frame of the fluoroscopic sequence. In the first step of the optimisation, only the first frame of the fluoroscopic sequence is used, and only the inplane rigid transformation parameters $\left(t_{x}, t_{z}, \beta\right)$ are estimated along with the global scale 
factor $\left(s_{\text {global }}\right)$ and the relative scale parameters of each bone $\left(s_{i}\right)$. The first significant parameter of the kinematic model $\left(b^{m}\right)$ is also estimated to provide an estimate of the overall pose. Other, less significant modes may include components of deviation along the out-of-plane direction that would affect the estimation of the global scale parameter. Inclusion of this first step resulted in significantly lower estimated error along the outof-plane direction than optimisation without this step. Starting from this initial estimate of pose, the first frame is evaluated again, taking all the parameters into account (except $\left.t_{y}\right)$ in the following sequence: $t_{x}, t_{z}, \beta, \alpha, \gamma, b^{m}, s_{\text {global }}, s_{i}$ and $b_{i}^{q}$. After convergence, the estimated pose of the current frame is used as the starting pose for the next frame. The shape model parameters $b_{i}^{q}$ are only estimated once in the first frame. From our initial experiments, the shape parameters are not improved significantly when we include more frames and the fitting is made significantly more complex and time consuming. At each stage, when $t_{x}, t_{z}, \beta, \alpha$ and $\gamma$ are estimated, only the region immediately surrounding the radius and ulna are used for cost function evaluation, while the larger region that includes the carpal bones is used for estimating the other parameters. There are about 60-80 frames per sequence. The whole process was performed in a 3-level multi-scale framework at each frame to enhance the robustness of the registration.

\section{Evaluation}

The ground truth of the recovered 3D pose corresponding to real fluoroscopic sequences is almost impossible to obtain. It would require the synchronisation of $3 \mathrm{D}$ imaging with the fluoroscopy. Hence, we evaluated our framework based on a number of simulated fluoroscopic sequences generated from the 3D CT data. All CT volumes have been resampled to an isocubic volume with voxel dimension of $0.5 \mathrm{~mm}$. We linearly interpolated a number of poses between the neutral pose and two extreme poses of radial-ulnar deviation in a full movement cycle containing 50 poses for each of 10 subjects. The ray-casting method was then used to generate a simulated fluoroscopic sequence from those interpolated 3D data. We evaluated the proposed framework in the leave-one-out manner. The 3D pose of the simulated test subject was then calculated as described in section 4, and registration error measured by the 3D Euclidian distance of each corresponding point of the mesh between the target pose and the estimated pose is presented in Table 1 The error of the registration is mainly caused by the ill posed problem (confusion between the scale and translation along the out-of-plane direction), whereas the errors along the in-plane directions are very small with average error of about 2 pixels and maximum error within 4 pixels.

It is important to mention that the relative positions of the carpal bones with respect to each other can be estimated much more accurately than the absolute positions of the individual bones. The registration error of the $3 \mathrm{D}$ distance between the centroid of Triquetrum and the centroid of Lunate (dTL), and the distance between the centroid of Lunate and the centroid of Scaphoid (dLS) were also measured. The errors are $1.18 \pm 0.74$ and $1.82 \pm 0.99$ pixels for dTL and dLS respectively, compared to a bone size of about 30 pixels. One of the conditions that may be assessed using this method is dissociations, where the distance between the bones is larger than normal. Scapholunate dissociation is one of the most common of these. We normalise the dLS by dividing it by the estimated global scale factor $s_{\text {global }}$ and an average of the scale factor $s_{i}$ for lunate and 
Table 1. The average error, measured in $3 \mathrm{D}$, between the target and estimated correspondence points of each carpal bone of 10 subjects: Triquetrum(Tri), Lunate(Lun), Scaphoid(Sca), Pisiform(Pis), Hamate(Ham), Capitate (Cap), Trapezoid (Trd) Trapezium (Trm). The measurement errors of dTL and dLS.

\begin{tabular}{|c|c|c|c|c|c|c|c|c|c||c|c|}
\hline & eTri & eLun & eSca & ePis & eHam & eCap & eTrd & eTrm & Total & eTL & eLS \\
\hline Err3D & $5.4 \pm 2.6$ & $5.1 \pm 2.5$ & $6.5 \pm 3.6$ & $6.8 \pm 3.7$ & $6.5 \pm 3.8$ & $6.6 \pm 4.0$ & $6.5 \pm 4.6$ & $7.6 \pm 4.3$ & $6.3 \pm 3.7$ & $1.18 \pm 0.74$ & $1.82 \pm 0.99$ \\
\hline ErrX & $1.6 \pm 1.3$ & $2.0 \pm 1.6$ & $2.1 \pm 1.8$ & $2.4 \pm 1.9$ & $1.8 \pm 1.4$ & $2.1 \pm 1.5$ & $1.8 \pm 1.4$ & $2.2 \pm 1.8$ & $2.1 \pm 1.7$ & $/$ & $/$ \\
\hline ErrY & $3.7 \pm 2.8$ & $3.0 \pm 2.6$ & $4.9 \pm 3.7$ & $4.8 \pm 3.9$ & $5.4 \pm 4.2$ & $5.3 \pm 4.4$ & $5.5 \pm 5.0$ & $6.1 \pm 4.6$ & $4.6 \pm 4.0$ & $/$ & $/$ \\
\hline ErrZ & $2.5 \pm 2.0$ & $2.5 \pm 1.9$ & $2.2 \pm 1.8$ & $2.6 \pm 2.1$ & $1.6 \pm 1.3$ & $1.7 \pm 1.3$ & $1.5 \pm 1.2$ & $2.2 \pm 2.0$ & $2.3 \pm 1.9$ & $/$ & $/$ \\
\hline
\end{tabular}
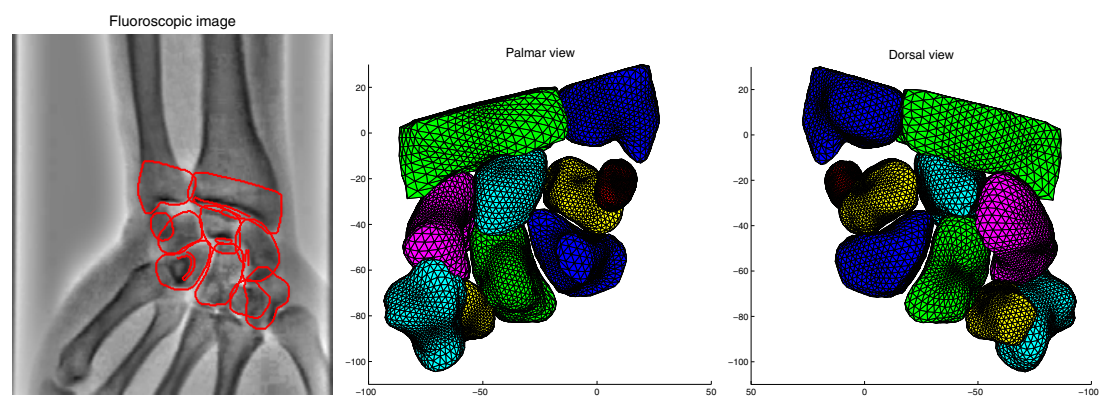

Fig. 3. Registration result of one frame from a real fluoroscopic sequence. The registration result for the whole sequence can be found in [11].

scaphoid. From the tested 10 subjects, we successfully identified the subjects suffering from scapholunate dissociation ( $\mathrm{dLS}=38.78 \pm 1.53$ pixels) from the normal subjects ( $\mathrm{dLS}=34.49 \pm 0.83$ pixels). Making this type of measurement without a $3 \mathrm{D}$ statistical model would be impossible.

We also tested our framework on real fluoroscopic sequences. Although the matching error cannot be quantified, the registration results show good visual correspondence and have been confirmed by a clinician. A sample frame of the matching result and the corresponding 3D pose are shown in Fig. 3 in which the projected contours from the 3D mesh model are superimposed on the preprocessed fluoroscopy image. The estimated 3D mesh model in the palmar and dorsal views are shown in middle and right respectively. The registration result for the whole sequence can be found in [11].

\section{Concluding Remarks}

We have presented a complete framework that is able to infer the $3 \mathrm{D}$ motion of carpal bones from a single view fluoroscopic sequence. It uses a hybrid statistical model to estimate both the kinematics and bone shapes from the fluoroscopic sequences allowing the motion of carpal bones during radial-ulnar deviation to be estimated. Particularly, the relative positions between carpal bones can be estimated accurately. This is potentially useful for detection of dissociation conditions, such as scapholunate dissociation, 
where the underlying pathology is a rupture of one or more ligaments, and the diagnosis rests on a judgement regarding the joint separation.

In further work we will extend the current statistical model with more training data (in progress) and test the framework for the flexion-extension movement.

\section{References}

1. Snel, J.G., Venema, H.W., Moojen, T.M., Ritt, M., Grimbergen, C.A., den Heeten, G.J.: Quantitative in vivo analysis of the kinematics of carpal bones from three-dimensional CT images using a deformable surface model and a three-dimensional matching technique. Medical Physics 27, 2037-2047 (2000)

2. Sonenblum, S.E., Crisco, J.J., Kang, L., Akelman, E.: In vivo motion of the scaphotrapeziotrapezoidal (STT) joint. Journal of Biomechanics 37, 645-652 (2004)

3. van de Giessen, M., Streekstra, G.J., Strackee, S.D., Maas, M., Grimbergen, K.A., van Vliet, L.J., Vos, F.M.: Constrained Registration of the Wrist Joint. IEEE Transactions on Medical Imaging 28(12), 1861-1869 (2009)

4. Foumani, M., Strackee, S.D., Jonges, R., Blankevoort, L., Zwinderman, A.H., Carelsen, B., Streekstra, G.J.: In-vivo three-dimensional carpal bone kinematics during flexion-extension and radio-ulnar deviation of the wrist: Dynamic motion versus step-wise static wrist positions. Journal of Biomechanics 42, 2664-2671 (2009)

5. Penney, G.P., Batchelor, P.G., Hill, D.L.G., Hawkes, D.J., Weese, J.: Validation of a two- to three-dimensional registration algorithm for aligning preoperative CT images and intraoperative fluoroscopy images. Medical Physics 28, 1024-1032 (2001)

6. Zheng, G.: Statistically deformable $2 \mathrm{D} / 3 \mathrm{D}$ registration for accurate determination of postoperative cup orientation from single standard X-ray radiograph. In: Yang, G., Hawkes, D., Rueckert, D., Noble, A., Taylor, C. (eds.) MICCAI 2009. LNCS, vol. 5761, pp. 820-827. Springer, Heidelberg (2009)

7. Chen, X., Graham, J., Hutchinson, C.E.: Integrated framework for simultaneous segmentation and registration of carpal bones. In: Accepted by the 18th ICIP, Belgium (2011)

8. Davies, R.H., Twining, C., Cootes, T.F., Taylor, C.J.: Building 3-D Statistical Shape Models by Direct Optimisation. IEEE Transactions on Medical Imaging 29(4), 961-980 (2010)

9. Amenta, N.: The Crust Algorithm for 3D Surface Reconstruction. In: Proceeding of the Fifteenth Annual Symposium on Computational Geometry (1999)

10. Black, M.J., Sapiro, G.: Edges as Outliers: Anisotropic Smoothing Using Local Image Statistics. In: Nielsen, M., Johansen, P., Fogh Olsen, O., Weickert, J. (eds.) Scale-Space 1999. LNCS, vol. 1682, pp. 259-270. Springer, Heidelberg (1999)

11. http://personalpages.manchester.ac.uk/staff/xin.chen/CarpalReg.htm 